\title{
Perspective
}

\section{Balancing treatment and side-effects in severe asthma: a patient and professional perspective}

\section{The patient perspective: Brenda Semple}

When my asthma is at its worst, my daily life is affected greatly. I cannot go for walks, which I adore, and stairs are a challenge (and as I live in a house, I usually have to pick a floor and stick to it!). Housework is a huge chore and, as a normally very social person, I find interaction exhausting. I was a full-time primary school teacher and had to medically retire at age 47 as my asthma exacerbations made continuity in my job impossible. I am prone to chest infections so I would feel pretty lousy and experience chest pain on top of the breathlessness.

Medications for my asthma vary according to how stable I am at the time. During flare-ups I could be on $40 \mathrm{mg}$ or more of prednisone a day. Whilst this is a lifesaving drug for me and, at times, I am often very grateful for it, it comes at a price.

I am often exhausted and struggle to stay awake during the day, but am wide awake at 03:00-04:00 and sleep after that is virtually impossible.

My emotions are all over the place. I can be irritable one moment and crying inexplicably the next. My family know the score by now, but as a generally happy-go-lucky person, this always takes me by surprise and then I feel guilty for acting out of character. I tend to hide away from people when I am on high doses, as a kind of self-protection. I don't like how I am at these times.

@ERSpublications

Oral corticosteroid side-effects in severe asthma affect a person's image and sense of self. Patients balance a web of risks and benefits around treatment adherence. Biological therapies may offer targeted treatment with a lower side-effect burden. https://bit.ly/3cHmcuk
I am prone to Cushing syndrome and my face can get very big as a result of the steroids. In the past, my body has also gotten pretty large, although I have learned how to curb the huge appetite and don't gain as much body weight as I used to. I have no control over the facial roundness or hair growth, however, which has a detrimental effect on my self-confidence. There is hardly a photograph of me with our kids growing up as I was always too conscious of being caught on camera with my "moon face". I always thought

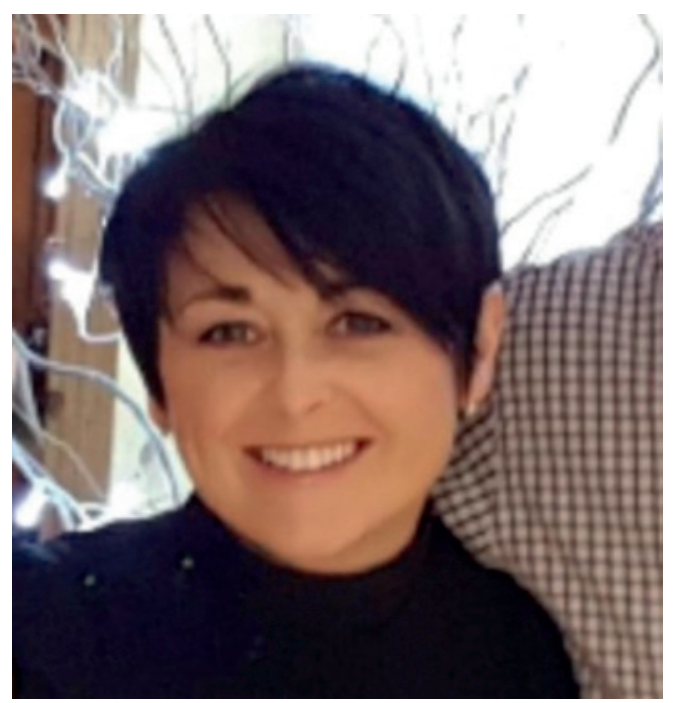

Brenda Semple.
Cite as: Semple B, Porsbjerg C, Coleman C. Balancing treatment and side-effects in severe asthma: a patient and professional perspective. Breathe 2021; 17: 210045. 
how unfair it was that it had to be my face; you can't hide your face with a baggy top!

I also have to take other medications too as a result of being on steroids. I take risedronate sodium and Adcal D3 to look after my bones, and pantoprazole to avoid heartburn and other stomach issues.

I receive a benralizumab injection every 8 weeks. Previous to this, I was on omalizumab injections, which meant I had to travel to the nearest severe asthma clinic every 2 weeks. This impacted on my job whilst I was working and involved a lot of travel because I live 55 miles away. These injections are life changing, however, and I would have moved mountains to get the quality of life which they promised and, in my case, delivered.

I don't think I have ever had a direct conversation with my severe asthma doctor about the weight gain caused by steroid treatment. I have on a few occasions gotten a bit emotional when it was apparent that oral steroids were my only option. I suppose l just assumed my doctor knew the reason why and, at that stage, I was in dire need of an intervention, so vanity took bottom place in the list for both of us.

As much as I would love an alternative treatment for exacerbations, there is none out there that I know of, so in a critical situation there really is no choice. Also, because of the nature of the drug, stopping it or lowering the dose when I feel better (and believe me, I have considered it, many, many times) is dangerous and self-sabotaging.

I have learned over the years how to be vigilant and control excess weight gain but not stop the steroid side-effects entirely.

I think doctors are fully committed to making their patients better. That is their job. They are not therapists. It would do no harm though, when prescribing high-dose steroids, to perhaps explain about the appetite increase, recommend a healthy diet, suggest appropriate exercise when symptoms decrease and reassure the patient that hopefully any changes in appearance will be short lived. Forewarned is forearmed, as they say.

\section{The professional perspective: Celeste Porsbjerg}

I frequently come across patients with sideeffects and body image changes in the severe asthma clinic. A lot of patients are put on oral corticosteroids in order to get their asthma under control, often for prolonged periods, and many have put on a lot of weight by the time they are referred to my clinic. As a severe asthma specialist, I can see that it is one thing getting a disease that makes your life difficult, but you also end up looking like someone else. I often get comments from patients such as "I don't look like myself", "I can't recognise myself", "I've become a different person".
There is an element of altering your selfunderstanding, your self-identity, when you get a disease: you lose something of yourself, part of your personality, part of who you are. When that is coupled with a change in your appearance, it can be very devastating and stigmatising. There has been little research in this area to understand patient perspectives and the balancing act between adherence and side-effects. A recent study by ApPS et al. [1] highlighted the impact on an individual: "I remember talking to my GP about my concerns and he said well the answer is simply you either want to breathe or you want to be slim, which would you rather have? [...] And it's like well breathe, you know, but it was really difficult to watch myself disappear with being on steroids [...] I lost my cheekbones, and I remember looking in the mirror one day and the only part of me I could recognise was my hair colour and my eye colour, and everything else about me I couldn't".

These patient experiences may offer part of the explanation for the fact that poor adherence is a relatively common phenomenon, even in patients with severe asthma: patients struggle to accept such severe side-effects, and may for periods opt to refrain from taking their full dose of medications. Having open conversations with our patients to understand their reasons for non-adherence, in a tolerant and empathetic manner, is key in the management of severe asthma.

My role as a severe asthma specialist is to help patients to best manage their disease. Luckily, we now have a range of new biological therapies in severe asthma which offer better asthma management, as well as reducing the burden of traditional treatments such as oral corticosteroids. In severe asthma, comorbidities are very important and should always be managed before starting on a biological therapy. Obesity can contribute to poor asthma control as it increases dyspnoea

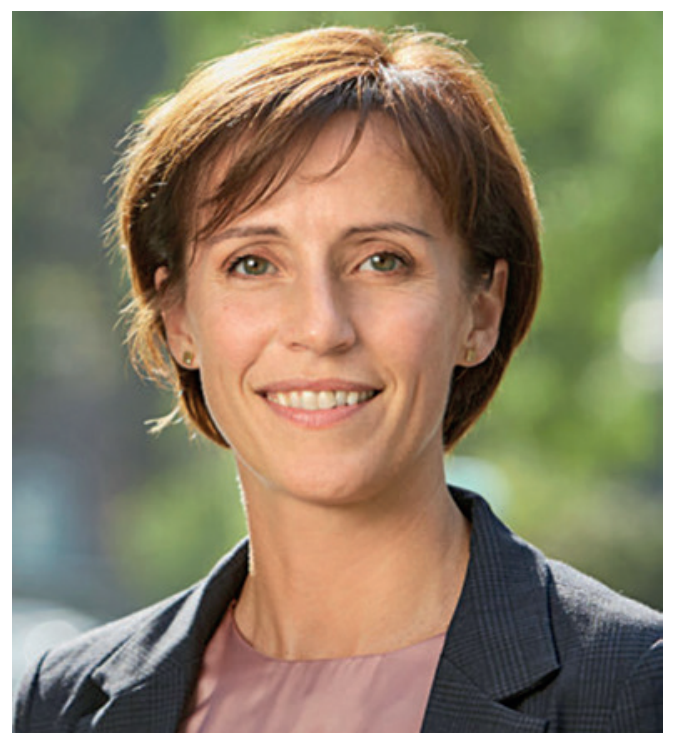

Celeste Porsbjerg. 
and inflammation. This can often lead to a vicious cycle of poor asthma control, steroid treatment and weight gain.

In my clinic we have a good dietitian. Evidence shows that even a minor level of weight loss can have a positive impact on asthma control [2], so we try to get patients into a positive circle: "I can lose weight, when I lose weight I feel better, I get more energy and I can work towards even more weight loss".

For many people, having severe asthma is devastating to their quality of life and the things they can do at work and with their family. Talking to patients about their weight or body image isn't a formal part of our routine assessment, but most good specialists should be talking about this in their clinical practice; it builds a picture of what it is like to be a person with this disease, not just a patient.

I believe that most severe asthma specialists already have a more holistic approach than mild or moderate asthma practice, and many doctors discuss these issues with severe asthma patients. I have worked closely with Prof. Vanessa McDonald in Australia and have been inspired by her research into quality of life and improving outcomes in severe asthma [3]: "your patient will have multiple problems, try and identify what they are and identify the one you should work with first, rather than trying to do everything at the same time. Work out what the individual person can achieve, rather than saying 'lose weight, start to exercise, do this and that all at the same time'". We must tailor our approaches to best support each individual. It is a different, more holistic approach. Normally, in milder asthma management, we would just focus on the lungs.

Access to a dietitian is not part of our routine care pathway, but it is available if a patient is overweight and might benefit from specialist support and, importantly, if we feel it is the appropriate time and the appropriate patient. Specialist mental health services, such as counselling or psychotherapy tailored for severe asthma patients, are not yet available in Denmark, although we are learning from other countries who have made progress in this area.

When patients are first referred to me, they are normally being considered for biological treatment and typically are "drowning" under a huge burden of symptoms and treatment. The topic of side-effects or weight gain isn't their first priority, but it is part of the wider picture they paint in terms of the impact on their identity and self-confidence - saying things like "I've lost myself", "I can't physically recognise myself".

Although some patients will raise it directly, we must be mindful that others will keep quiet and we as health professionals must make sure it isn't overlooked. The change in physical appearance as a result of treatment side-effects is clearly something patients don't feel comfortable with. It almost seems like a shameful thing for them. However, it is not just about body image, it is also changes in the individual's personality, being irritable, you become a difficult version of yourself. As a doctor, it's not so much if I see a patient who is overweight, it's the combination - red, agitated, not at ease then we start talking about it.

And it's important to talk about it. I imagine patients think there is no alternative. Doctors are probably not very conscious of it, and may feel they aren't able to help - in a busy clinic, do you really want to ask about problems that you can't fix?

In Denmark we try to avoid prescribing high-dose oral corticosteroids. In practice, we tend to give very high doses of inhaled steroids, and likely see fewer patients with weight gain and other side-effects as a result. Psychologically, doctors and patients seem to perceive that a tablet form of treatment means their asthma is "bad" and prefer an inhaler. Part of that is the potential side-effects. Sometimes this goes against the guidelines; there are certainly no studies to support this approach.

Now, with the development of biological medications, there is really no excuse to treat patients with oral steroids. Hopefully in the future we will see fewer patients on long-term steroids, including some who will start biological treatment sooner and will never need steroids. Most countries have national criteria for accessing high-cost biological treatment. Sometimes this means that patients must use oral corticosteroids a certain number of times each year before they can be referred for a biological treatment, and some national guidelines, such as in Finland, state that patients who respond to oral steroids cannot be offered biological treatment. There are also other areas in Europe with limited access to biological therapies, and many patients will not be offered biological treatment. Often, patients do not get referred by their primary care doctor to a specialist severe asthma clinic; one possible explanation is that when they get treated for an exacerbation with a course of oral steroids, they may respond well to steroids if the patient has type 2, or "allergic", asthma. Neither the patient nor the doctor may realise that for patients with repeated exacerbations, there are better treatment alternatives nowadays. In Denmark today, we have between 800 and 900 patients on biological treatment. However, we estimate that there are 10000 patients who should be on a biological treatment - there is still a high level of avoidable overtreatment with oral steroids.

As a severe asthma clinician, I must balance the guidelines and the reality of the patient in front of me: the guidelines state that they should lose weight before starting a biological treatment, but if a patient is stuck on prednisone and still has asthma symptoms as well as side-effects, then they are unlikely to be able to lose enough weight to start a biological therapy. In those cases, I believe it is indicated to start the patient on biological treatment and then work with the comorbidities when they have more energy and a lower symptom burden. 
Nevertheless, overall, times are changing and we are hopefully moving away from the approach of treating everything with steroids. I hope that a modern-day severe asthma clinician does not put patients on oral corticosteroids. The message for patients is: if your doctor is suggesting an oral steroid, you should ask why you're not being offered a biological. If you don't fit the criteria because of the type of asthma you have (non-eosinophilic), then you probably will get nothing but harm from oral steroids in the long term. For doctors: if you ever think about prescribing regular oral corticosteroids for asthma, think twice - there are other options. For those practising in areas where biological therapies are not yet available: consider how you can best support your patient to manage the side-effects of treatment through tailored dietary and exercise support.

\section{Conclusion}

The side-effects of oral corticosteroid treatment in severe asthma can have a devastating impact on the person's self-identity, confidence and overall quality of life. Patients balance the need for life-saving treatment with the negative impact on their sense of self, and healthcare professionals have an important role to play in holistic care. New treatments for severe asthma offer the possibility of reducing reliance on oral corticosteroid treatment, and thus reducing the side-effect burden on patients.

\section{Affiliations}

Brenda Semple ${ }^{1}$, Celeste Porsbjerg ${ }^{2}$, Courtney Coleman ${ }^{1}$

${ }^{1}$ European Lung Foundation, Sheffield, UK. ${ }^{2}$ Respiratory Research Unit, Dept of Respiratory Medicine, Bispebjerg and Frederiksberg Hospital, Copenhagen, Denmark.

\section{Conflict of interest}

B. Semple has nothing to disclose. C. Porsbjerg has received grants or contracts from AstraZeneca, GSK, Teva, Sanofi, Pharmaxis, Chiesi, MSD and Novartis, consulting fees from AstraZeneca, GSK, Teva, Sanofi, Chiesi and Novartis, and payment or honoraria for lectures, presentations, speakers bureaus, manuscript writing or educational events from AstraZeneca, GSK, Teva, Sanofi, Chiesi and Novartis, in the past 36 months. C. Coleman is an employee of the European Lung Foundation.

\section{References}

1. Apps LD, Chantrell S, Majd S, et al. Patient perceptions of living with severe asthma: challenges to effective management. J Allergy Clin Immunol Pract 2019; 7: 2613-2621.

2. Dias-Júnior SA, Reis M, de Carvalho-Pinto RM, et al. Effects of weight loss on asthma control in obese patients with severe asthma. Eur Respir J 2014; 43: 1368-1377.
3. Hiles SA, Gibson PG, Agusti A, et al. Treatable traits that predict health status and treatment response in airway disease. J Allergy Clin Immunol Pract 2021; 9: 1255-1264. 